Mittheilungen aus dem ohemischen Laboratorium der Universität Halle $a_{1} \mathrm{~S}$.

\title{
Kohlenstoffdoppelbindung und Carbonyl;
}

\author{
von $D$. Vorlünder.
}

Dass Kohlenstoffdoppelbindungen sich oft verhalten wie Carbonyle in Aldehyden und Ketonen ist durch ältere und neuere Versuche festgestellt. Gleichwohl giebt es auch Fälle, besonders Additionsvorgänge, bei welchen die Kohlenstoffdoppelbindung und das Carbonyl verschieden reagiren. Das Carbonyl addirt zumeist sehr leicht Ammoniak, Hydroxylamin, Cyanwasserstoff, Malonester, Acetessigester u. a., während die Kohlenstoffdoppelbindung, z. B. im Aethylen, Vinylbromid, Stilben u. a. mit denselben Substanzen nicht leicht reagirt. Dagegen bildet die Kohlenstoffdoppelbindung mit Halogen, Halogenwasserstoff, unterchloriger Säure, Nitrosylchlorid u. dergl. beständige Additionsproducte und nicht das Carbonyl.

Eine auffällige Erscheinung zeigt sich nun, wenn die Kohlenstoffdoppelbindung und das Carbonyl in einer Verbindung rereint sind. Jene Agentien, Ammoniak, Hydroxylamin, Malonester werden von der Kohlenstoffdoppelbindung, die für sich allein kaum reactionsfähig ist, aufgenommen, sobald neben derselben ein Carbonyl steht, wie in $\alpha$-ungesättigten Säureestern und Ketonen mit der Gruppe $\mathrm{C}=\mathrm{C}-\mathrm{C}=0$. In diesen $\alpha$-ungesättigten Verbindungen ist sogar die Kohlenstoffdoppelbindung unter Umständen reactionsfähiger als das Carbonyl, was $\mathrm{H}$ arries ${ }^{1}$ ) durch Anlagerung von Hydroxylamin und Wasserstoff nachgewiesen hat, und was man im Allgemeinen aus dem Verhalten der Verbindungen gegen Ammoniak, Alkohol, Cyanwasserstoff ${ }^{2}$ ),

\footnotetext{
1) Ber. d. deutsch. chem. Ges. 29, 380; 30, 230 und $2726 ; \mathbf{3 1}$, 1371 ; $\mathbf{3 2}, 1315 \mathrm{ff}$; diese Annalen $\mathbf{2 9 6}, 295$.

2) Bredt and Kallen, diese Annalen $\mathbf{2 9 3}, 338$.
} 
Aceton $^{8}$ ), Acetophenon ${ }^{4}$ ), Bernsteinsäureester ${ }^{5}$ ), Malonester ${ }^{6}$ ), Acetessigester ${ }^{6}$ ) u. s. w. schliessen kann. Diese Substanzen der Form RH reagiren mit $\alpha$-ungesättigten Verbindungen in der Weise, dass Wasserstoff an den $\alpha$-Kohlenstoff, der übrige Rest an den $\beta$-Kohlenstoff der ungesättigten Verbindung tritt; der Vorgang ist wie fast alle Additionsvorgänge umkehrbar ${ }^{7}$ ).

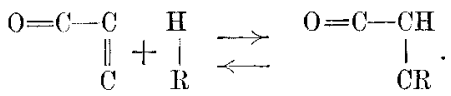

\section{Die Combination $\mathrm{C}-\mathrm{C}-\mathrm{C}=\mathrm{O}$.}

Die auffallenden Eigenschaften der $\boldsymbol{\alpha}$-ungesättigten Ester und Ketone können in befriedigender Weise aus den üblichen Formeln abgeleitet werden, wenn man das Verhalten anderer carbonylhaltiger Verbindungen berücksichtigt, und die Combinationen

$$
\begin{array}{r}
\mathrm{C}-\mathrm{C}-\mathrm{C}=\mathrm{O} \\
\mathrm{H}-\mathrm{C}-\mathrm{C}=\mathrm{O} \\
\mathrm{H}-\mathrm{O}-\mathrm{C}=0 \\
\mathrm{Cl}-\mathrm{C}=0
\end{array}
$$

mit einander vergleicht.

1) Elemente oder Elementgruppen, welche dem Carbonyl benachbart sind, haben im Allgemeinen einen vollständig anderen Charakter als ohne das Carbonyl. Zwischen einem Alkohol $(\mathrm{HO}) \mathrm{CH}_{2}$ und einer Säure $(\mathrm{HO}) \mathrm{C}=0$ besteht ein so grosser Unterschied, dass man das Hydroxyl des Alkohols in der Carbonsäure kaum wiederzuerkennen vermag; ähnlich ergeht es dem Chlor oder Brom bei dem Uebergange von Halogenalkyl $\mathrm{Cl}_{-} \mathrm{CH}_{2}$ zum Halogenacyl $\mathrm{Cl}-\mathrm{C}=0$. Aber auch das Car-

3) Bredt und Rübel, diese Annalen $\mathbf{2 9 9}, 184$.

4) v. Kostanecki, Ber. d. deutsch. chem. Ges. 29, 240, 1488 und 2245 ; Sto b be, Ber. d. deutsch. chem. Ges. B4, 604 .

5) Diese Amalen B14, 113.

6) Claisen and Komnenos, diese Amalen 218, 160; Michacl, Journ. f. pract. Chem. $[2] \mathbf{3 5}, 349 ; \mathbf{4 5}, 390$.

7 Ber. d. deutsch. chem. Ges. 33, 3185. 
bonyl erleidet eine wesentliche Veränderung durch Vertauschung der benachbarten Elemente. Die Additions- und Condensationsfähigkeit des Carbonyls der Aldehyde und Ketone verschwindet in dem Maasse, als die dem Carbonyl benachbarten Elemente, Chlor, Hydroxyl in Säurechloriden und Säuren beweglicher werden $(\text { Thiele })^{8}$ ). Daraus folgt, dass die Kohlenstoffdoppelbindung in $\alpha$-ungesättigten Verbindungen mit der Combination $\mathrm{C}-\mathrm{C}-\mathrm{C}=0$ andere Eigenschaften haben und reactionsfähiger sein muss, als ohne das Carbonyl z. B. in Aethylen und Styrol, und dass umgekehrt das Carbonyl nach seiner Vereinigung mit der Kohlenstoffdoppelbindung die Aldehyd- und Ketonreactionen in weit geringerem Grade zeigt, als vorher.

2) Bei den meisten Umwandlungen der Sänrechloride oder Carbonsäuren reagirt nicht das Carbonyl, sondern das Chlor bezw. der Sauerstoff und Wasserstoff des Hydroxyls. Demnach ist auch bei $\alpha$-ungesättigten Verbindungen die Kohlenstoffdoppelbindung der leichter reagirende Theil der Combination

$$
\mathrm{C}=\mathrm{C}-\mathrm{C}=\mathrm{O} \text {, }
$$

welche den Combinationen

entspricht.

$$
\begin{aligned}
\mathrm{H}-\mathrm{O}-\mathrm{C} & =0 \\
\mathrm{Cl}-\mathrm{C} & =\mathrm{O}
\end{aligned}
$$

Die hier verglichenen Gruppen sind natürlich insofern verschieden, als $\mathrm{C}=\mathrm{C}, \mathrm{Cl}$ und $\mathrm{HO}$ sich von einander unterscheiden.

3) Durch Verbindung mit dem Carbonyl ist der Gegensatz zwischen dem Wasserstoff und Sauerstoff des IIydroxyls ausserordentlich gesteigert: $\stackrel{+}{\mathrm{H}}-\mathrm{C}-\mathrm{C}=\mathrm{O}$. Eine ähnliche, wenn auch dem Charakter des Kohlenstoft's entsprechend geringere Steigerung wird in der Combination $\stackrel{+}{\mathrm{C}}=\overline{\mathrm{C}}-\mathrm{C}=0$ eintreten; ein Addend $\stackrel{+}{\mathrm{H}} \mathrm{R}$ giebt wegen dieses positiven und negativen Gegensatzes mit der Kohlenstoffdoppelbindung das Additionsproduct

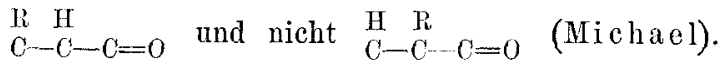

\footnotetext{
S) Diese Amalen :306, 116.
} 
4) Die Reactionsfähigkeit der Kohlenstoffdoppelbindung in der Combination $\mathrm{C}=\mathrm{C}-\mathrm{C}=\mathrm{O}$ wird durch Hinzutritt eines zweiten Carbonyls<smiles>C=C(C=O)C=O</smiles>

in ähnlicher Weise vergrössert, wie die Reactionsfähigkeit des $\mathrm{HC}$ in<smiles>O=CC=O</smiles>

(vergl. Zimmtsäureester und Benzalmalonester mit Essigester und Malonester).

5) In weiterer Entfernung vom Carbonyl hört fast jede Beziehung zum Hydroxyl, Chlor oder $\mathrm{HC}$ auf:

$$
\begin{array}{rlrl}
\mathrm{HO}-\mathrm{CH}_{2}-\mathrm{C}=\mathrm{O} & \mathrm{Cl}-\mathrm{CH}_{2}-\mathrm{C}=\mathrm{O} & \mathrm{H}_{3} \mathrm{C}-\mathrm{O}-\mathrm{C}=0 \\
\mathrm{HO}-\mathrm{C}=\mathrm{Cl}-\mathrm{C}=\mathrm{O} & \mathrm{H} & \mathrm{H}_{3} \mathrm{C}-\mathrm{C}=0
\end{array}
$$

so dass die Gruppen $\mathrm{HO}, \mathrm{Cl}$ u. a. den gewöhnlichen Charakter des Alkohols, Halogenalkyls u. a., aber nicht mehr den der Carbonsäure und des Säurechlorids zeigen. Die gleiche Erscheinung findet sich bei der Kohlenstoffdoppelbindung; denn ungesättigte Verbindungen, in denen die Kohlenstoffdoppelbindung dem Carbonyl nicht benachbart ist, wie Diallylmalonester, Allylaceton, Phenyl- $\beta \gamma$-crotonsäureester

$$
\begin{array}{r}
\mathrm{C}=\mathrm{C}-\mathrm{CH}_{2}-\mathrm{CH}_{2}-\mathrm{C}=0 \\
\mathrm{C}=\mathrm{C}-\mathrm{CH}_{2}-\mathrm{C}=0 \\
\mathrm{C}=\mathrm{C}-\mathrm{C}=\mathrm{O}
\end{array}
$$

haben auch nicht das typische Additionsvermögen der $\alpha$-ungesättigten Säureester und Ketone.

6) Da der Einfluss des Carbonyls kaum über das dritte Element der Combination

$$
\stackrel{2}{\mathrm{E}}-\stackrel{2}{\mathrm{H}}-\stackrel{\mathrm{C}}{\mathrm{C}}=\mathrm{O} \quad \text { (z. B. } \mathrm{H}-\mathrm{O}-\mathrm{C}=\mathrm{O} \text { oder } \mathrm{H}-\mathrm{C}-\mathrm{C}=\mathrm{O} \text { ) }
$$

hinausreicht (vergl. 5), so ist zunächst zu erwarten, dass der Addend $\stackrel{+-}{\mathrm{H}}$ bei der Vereinigung mit einer Kette von Kohlenstoffdoppelbindungen

$$
\begin{aligned}
\mathrm{C}=\mathrm{C} \cdot \mathrm{C}=\mathrm{C}-\mathrm{C}=\mathrm{O} \\
\mathrm{H}-\mathrm{O}-\mathrm{C}=\mathrm{O}
\end{aligned}
$$


mit der $\alpha \beta$-Doppelbindung reagirt. Thatsächlich ist nachgewiesen worden, dass das Keton

$$
\mathrm{C}_{6} \mathrm{H}_{5}-\mathrm{CH}=\mathrm{CH}-\mathrm{CH}=\mathrm{CH}-\mathrm{COCH}_{3}
$$

nicht nach der Zahl der Doppelbindungen zwei Mol. Malonester aufnimmt, sondern ein Mol., und zwar dem Charakter des Additionsproductes zufolge an $\alpha \beta$-Stellung ${ }^{9}$ ). Auch Cyanwasserstoff lagert sich nach den Untersuchungen ron $\mathrm{T}$ hiele und Meisenheimer ${ }^{10}$ ) an die $\alpha \beta$-Doppelbindung des Cinnamylidenmalonesters an:<smiles>CCOC(=O)C=CC(C#N)C=Cc1ccccc1</smiles>

Da jedoch die Carbonylwirkung in den Combinationen

$$
\begin{aligned}
& \mathrm{H}-\mathrm{O}-\mathrm{C}=\mathrm{C}-\mathrm{C}=\mathrm{O} \\
& \mathrm{H}-\mathrm{C}-\mathrm{C}=\mathrm{C}-\mathrm{C}=\mathrm{O}
\end{aligned}
$$

auf das $\mathrm{H}-\mathrm{O}$ bez. $\mathrm{H}-\mathrm{C}$ übertragbar ist, so muss in der analogen Combination

$$
\mathrm{C}=\mathrm{C}-\mathrm{C}=\mathrm{C}-\mathrm{C}=\mathrm{O}
$$

unter Umständen auch die $\gamma \delta$-Kohlenstoffdoppelbindung reactionsfähig sein. Dem entsprechend haben Thiele und Meis e nheimer bei der Anlagerung von Blausäure an Cinnamylidenmalonester und nach Verseifung des Nitrilesters eine Tricarbonsäure, wahrscheinlich

erhalten.

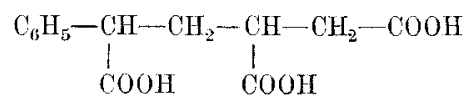

7) Ersetzt man das Carbonyl durch eine Kohlenstoffdoppelbindung, so erhält man aus einem Säurechlorid $\mathrm{Cl}-\mathrm{C}=0$ eine Verbindung mit $\mathrm{Cl}-\mathrm{C}=\mathrm{C}$, z. B. Vinylchlorid bezw. Vinylbromid, in welchem die Halogene nicht mehr so leicht wie im Säurechlorid reagiren; auch die Condensationsfähigkeit des Metbylenwasserstoffs in der Gruppe $\mathrm{H}_{2} \mathrm{C}-\mathrm{C}=\mathrm{O}$ lässt sich durch Vertauschung des Carbonyls mit der Kohlenstoffdoppelbindung zu $\mathrm{H}_{2} \mathrm{C}-\mathrm{C}=\mathrm{C}$

9) Diese Anualen $\mathbf{2 9 4}, 298$.

10) Diese Annalen B06, 247. 
nicht erzielen $\left.{ }^{11}\right)$. Dass ebenso die $\alpha$-ungesättigten Verbindungen, in denen das $\mathrm{C}=0$ durch $\mathrm{C}=\mathrm{C}, \mathrm{C}=\mathrm{N}$ oder $\mathrm{C}_{6} \mathrm{H}_{\tilde{5}}$ ersetzt ist,

$$
\begin{aligned}
& \mathrm{C}=\mathrm{C}-\mathrm{C}=\mathrm{C} \\
& \mathrm{C}=\mathrm{C}-\mathrm{C}=\mathrm{C} \\
& \mathrm{C}=\mathrm{C}-\mathrm{C}=\mathrm{N}
\end{aligned}
$$

ihr Additionsvermögen einbüssen, wird in der unten folgenden Untersuchung über das Verhalten ungesättigter Verbindungen gegen Natriummalonester nachgewiesen.

Somit ist der Vergleich der verschiedenartigen carbonylhaltigen Gruppen bis in die Einzelheiten formell durchführbar und man kann behaupten, dass diejenigen Eigenschaften des Carbonyls, welche das Chlor in Säurechloriden und den Wasserstoff in Carbonsäuren oder Ketomethanderivaten reactionsfähig machen, auch das Additionsvermögen der Kohlenstoffdoppelbindung in $\alpha$-ungesättigten Verbindungen hervorrufen.

Im Gegensatze hierzu sind nach der Theorie der Partialvalenzen von Thi ele nicht die Kohlenstoffdoppelbindungen $\mathrm{C}=\mathrm{C}$, sondern die Enden der Combination $\mathrm{C}=\mathrm{C}-\mathrm{C}=\mathrm{O}$ reactionsfähig

$$
\mathrm{C}=\mathrm{C}-\mathrm{C}=\mathrm{O}+\mathrm{HR}=\stackrel{\mathrm{C}}{\mathrm{C}}-\mathrm{C}=\mathrm{C}-\mathrm{O} \text {. }
$$

Wegen der Verwandtschaft des Wasserstoffs zum Sanerstoff ${ }^{12}$ ) sollen zunächst Enole entstehen, welche sich dann nach

11) Der Wasserstoff der Gruppe $\mathrm{C}=\mathrm{C} . \mathrm{CH}_{2}$ ist bei Anwendung allialischer Condensationsmittel nur dam leicht beweglich, wemm ausser der Kohlenstoffdoppelbindung andere ungesättigte Gruppen vorhanden sind, z. B. Carbonyl in $\mathrm{O}=\mathrm{C}-\mathrm{C}=\mathrm{C}-\mathrm{CH}_{2}$ (Henrich, Ber. d. deutsch. chem. Ges. B1, 2103), ungesättigter Stickstoff nnd Ring im Chinaldin (W. Wislicenus, Ber. d. dentsch. chem. Ges. BO, 1479; 33, 771), Nitryl im Nitrotoluol (Reissert, Ber. d. dentsch. chen. Ges. 30, 1030), Kohlenstoffing und Doppelbindungen im Cyklopentadiën (Thiele, Ber. d. deutsch. chem. Ges. B3, 666). In Gegenwart von sauren Condensationsmitteln ändert sich sowohl das Additionsvermögen der Kohlenstoffoppelbindung, als auch die reactive Wirkung anf benachbarten Wasserstoff.

12) Diese Amalen :306, 105 . 
bekannten Analogien in Ketone umlagern. Bei aller Bewunderung der Untersuchungen Thiele's vermag ich dieser Auffassung nicht zuzustimmen. Die hypothetischen Enolzwischenproducte scheinen mir keine Erklärung, sondern mehr eine Verwickelung des Gegenstandes zu bringen, da sie für das Verständniss nicht durchaus erforderlich sind. Ich glaube auch, dass der Wasserstoff nicht nothwendig zum Sauerstoff des Carbonyls wandern muss, sondern möchte aus den oben genannten Gründen dem Wasserstoff des Recht belassen, sich an den Kohlenstoff anzulagern:

$$
\begin{aligned}
& \mathrm{R} \\
& \mathrm{C}-\mathrm{C}-\mathrm{C}=\mathrm{O}
\end{aligned}
$$

Gegen die Auffassung spricht, dass die Verbindungen RH nicht an die Enden treten

$$
\stackrel{\mathrm{R}}{\mathrm{C}}=\mathrm{C}-\mathrm{C}=\mathrm{C}-\mathrm{C}=\stackrel{\mathrm{H}}{\mathrm{O}},
$$

was die Theorie unbedingt verlangt, sondern an die Mitte des Systems ${ }^{13}$ ) (vergl. 6). Demnach betrachte ich die ältere Formulirung der Additionsreactionen der $\alpha$-ungesättigten Ester und Ketone als die bessere, ohne die Bedeutung der Partialvalenzentheorie auf anderen Gebieten verkennen zu wollen.

\section{Die sogenannte negative Wirkung des Carbonyls.}

Erklärungen für das verschiedene Verhalten der Kohlenstoffdoppelbindung, des Carbonyls und der Combination beider hat man ausser in der Sonderstellung der Combination $\mathrm{E}=\mathrm{E}-\mathrm{E}=\mathrm{E}$ hauptsächlich darin gesucht, dass das Carbonyl ein stark negatives Radical sei; denn man kann das verschiedene Additionsvermögen von $\mathrm{C}=\mathrm{C}$ und $\mathrm{C}=0$ kaum anders als mit der verschieden negativen Natur von Kohlenstoff und Sauerstoff erklären.

Ich habe nun versucht, die negative Natur des Carbonyls zu bestimmen, indem ich der negativen Wirkung cine entgegengesetzt gerichtete, positive Wirkung gleichsam als Maassstab

13) Diesen Widerspruch mit der Theorie hat Thiele bereits hervorgehoben; dicse Annalen $\mathbf{B 0 6}, 106$. 
gegenüberstellte $\left.{ }^{14}\right)$. Wenn der Einfluss des Carbonyls in der Combination $\mathrm{C}=\mathrm{C}-\mathrm{C}=\mathrm{O}$ auf der negativen Natur des $\mathrm{C}=0$ beruht, so müsste er durch benachbarte positive Radicale, welche diese negative Beschaffenheit neutralisiren, abgeschwächt werden und zwar um so mehr, je stärker positiv oder basisch die Radicale sind. Steht das Carbonyl zwischen dem basischen Rest und der reactionsfähigen Kohlenstoffdoppelbindung, z. B. in den Särcreamiden

$$
\text { Alkyl- }[\mathrm{CH}=\mathrm{CH}]-\mathrm{C}=\mathrm{O}\left[\mathrm{NR}_{2}\right] \text {, }
$$

so kömnte die Reactionsfähigkeit abhängig sein von der Stärke der basischen Eigenschaften des in dem Säureamid enthaltenen Ammoniak- oder Aminrestes $\mathrm{NR}_{2}$.

Diese Anschauungsweise, welche auf Grund der üblichen Ansichten über die positive und negative Natur der Radicale wohl berechtigt ist, habe ich einer experimentellen Prüfung unterzogen. Eine Reihe von Additionen wurden unter gleichen Bedingungen ausgefübrt mit Natriummalonester ${ }^{15}$ ) und Zimmtsäureamiden, die den oben gestellten Bedingungen entsprechend gebaut sind:

$$
\begin{aligned}
\mathrm{C}_{6} \mathrm{H}_{5}-\mathrm{CH}=\mathrm{CH} & -\mathrm{CO}-\mathrm{NH}_{2} \\
& -\mathrm{CO}-\mathrm{NHCH}_{3} \\
& -\mathrm{CO}-\mathrm{NHC}_{2} \mathrm{H}_{5} \\
& -\mathrm{CO}-\mathrm{NHC}_{6} \mathrm{H}_{5} \\
& -\mathrm{CO}-\mathrm{N}\left(\mathrm{CH}_{3}\right)_{2} \\
& -\mathrm{CO}-\mathrm{N}_{2}\left(\mathrm{C}_{2} \mathrm{H}_{5}\right)_{2} \\
& -\mathrm{CO}-\mathrm{NC}_{5} \mathrm{H}_{10} \\
& -\mathrm{CO}-\mathrm{N}^{2}\left(\mathrm{C}_{6} \mathrm{H}_{5}\right)_{2} .
\end{aligned}
$$

Die Verbindungen haben mit Ausnahme des Anilids und Diphenylamids deutlich basische Eigenschaften. Die basischen Reste $\mathrm{NR}_{2}$ leiten sich vom Ammoniak, von primären und secun-

14) Die Untersuchung wurde zuerst vor drei Jahren in den Abhandl. d. Naturforsch. Ges. Halle 21, 23 ó veröffentlicht; vergl. Chem. Centralbl. 1899, I, 729.

$\left.{ }^{15}\right)$ Malonsäureester reagirt leicht und glatt mit der Kohlenstoffdoppelbindung $\alpha$-ungesüttigter Säureester und Ketone, ohne deren Carbonyl zи berühren. 
dären Aminen ab, deren ,Stärke“" sich auf Grund der elektrischen Leitfähigkeit wie folgt angeben lässt ${ }^{16}$ ) (Dissociationsconstante des Ammoniumhydroxyds $=1$ ):

$\begin{array}{lr}\text { Ammoniak } & 1,0 \\ \text { Methylamin } & 21,7 \\ \text { Aethylamin } & 23,0 \\ \text { Dimethylamin } & 32,2 \\ \text { Diäthylamin } & 52,6 \\ \text { Piperidin } & 68,7 .\end{array}$

Das Ammoniak ist unter den genannten die schwächste Base, das Piperidin die stärkste. Anilin ist ungefähr $10^{8} \mathrm{mal}$ schwächer als Ammoniak ${ }^{17}$ ). Die basischen Eigenschaften des Diphenylamins, welches noch viel schwächer ist als Anilin in Zahlen lässt sich dies nicht ausdrücken -- dürften demnach verschwindend gering sein im Vergleich mit den aliphatischen Basen. Hier könnte also ein Unterschied im Additionsvermögen der entsprechenden Zimmtsäurederivate besonders auffallend werden.

Zum Vergleich mit den Amiden diente der Zimmtsäureäthylester, dessen Additionsproducte mit Malonester bereits früher von $\mathrm{Michael^{18 }}$ ) sowie von Avery und Bouton ${ }^{19}$ ) beschrieben worden sind. Unsere Versuche mit Zimmtsäureamiden haben Folgendes ergeben:

Die Amide verhalten sich ganz analog wie der Ester; die Reaction mit Malonester verläuft in der gewöhnlichen Weise; z. B. mit Zimmtsäurepiperid

$$
\begin{gathered}
\mathrm{C}_{6} \mathrm{H}_{5} \cdot \mathrm{CH}=\mathrm{OH}-\mathrm{CO} . \mathrm{NC}_{5} \mathrm{H}_{10} \\
\underset{\mathrm{CH}}{\mathrm{CH}_{2}}\left(\mathrm{COOC}_{2} \mathrm{H}_{5}\right)_{2}
\end{gathered}=\underset{\mathrm{CH}\left(\mathrm{COOC}_{2} \mathrm{H}_{5}\right)_{2}}{\mathrm{C}_{5} \mathrm{H}_{5} \cdot \mathrm{CH}-\mathrm{CH}_{2}-\mathrm{CONC}_{5} \mathrm{H}_{10}}
$$

Der Diäthylester verwandelt sich durch Verseifung mit kalter alkoholischer Kalilauge in die Dicarbonsäure,

\footnotetext{
16) Bredig, Zeitschr. f. physikal. Chem. 13, 294; Ber. d. deutsch. chem. Ges. 31, 1633.

17) Bredig, Zeitschr. f. plysikal. Chem. 11, 830; 13, 306.

18) Journ. f. pract. Chem. [2] 35, 352.

19) Amer. chem. Journ. 20, 509; Chem. Centralbl. 1898, II, 274.
} 


$$
\begin{gathered}
\mathrm{C}_{6} \mathrm{H}_{5} \cdot \mathrm{CH}-\mathrm{CH}_{2}-\mathrm{CO} . \mathrm{NC}_{5} \mathrm{H}_{10} \\
\stackrel{\mathrm{CH}}{\mathrm{in}}(\mathrm{COOH})_{2}
\end{gathered},
$$

welche durch Kohlensäureabspaltung in die Monocarbonsäure

$$
\begin{aligned}
& \mathrm{C}_{6} \mathrm{H}_{5} \cdot \mathrm{CH}-\mathrm{CH}_{2}-\mathrm{CONC}_{6} \mathrm{H}_{10} \\
& \stackrel{\mathrm{CH}_{2}}{\mid}-\mathrm{COOH}
\end{aligned}
$$

übergeht. Diese zerfällt beim Kochen mit Salzsäure in Phenylglutarsäure und Piperidin.

Beim Piperid wurden die Zwischenproducte mit Ausnahme des Diäthylesters rein dargestellt. Beim Zimmtsäurediphenylamid war dies nicht möglich, da der zuerst entstehende Ester schon beim Verseifen mit kalter Kalilauge das Diphenylamin verliert.

Zimmtsäureamid und Anitid erleiden bei der Anlagerung von Malonsäureester eine innere Condensation, wahrscheinlich in folgender Weise:

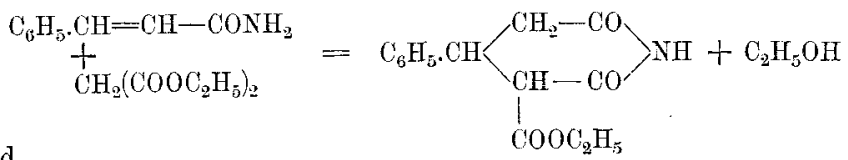

und

$$
=\mathrm{C}_{6} \mathrm{H}_{5} \cdot \mathrm{CH}\left\langle\begin{array}{c}
\mathrm{CH}_{\mathrm{CH}}-\mathrm{CO} \\
\mathrm{COOC}_{2} \mathrm{H}_{5}
\end{array} \mathrm{NC}_{6} \mathrm{H}_{5}+\mathrm{C}_{2} \mathrm{H}_{5} \mathrm{OH} .\right.
$$

Aus dem Anilester geht unter dem Einfluss kalter alkoholischer Kalilauge Phenylglutaranildicarbonsäure und nach der Kohlensäureabspaltung Phenylglutaranilsäure

$$
\mathrm{C}_{6} \mathrm{H}_{5} . \mathrm{CH}\left\langle\begin{array}{l}
\mathrm{CH}_{2}-\mathrm{CONHC}_{6} \mathrm{H}_{5} \\
\mathrm{CH}_{2}-\mathrm{COOH}
\end{array}\right.
$$

hervor, welche auch aus Phenylglutarsäureanbydrid und Anilin gewomnen wird:

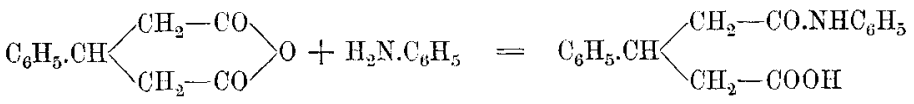

Die unverändert gebliebenen Zimmtsänreamide und die nach der Verseifung mit kalter alkoholischer Kalilauge zu ge- 
winnende Zimmtsäure wurde durch Wägung bestimmt, ein Verfahren, welches zwar nicht genau ist, aber immerhin ein Bild ron dem Verlaufe der Reaction giebt; die erhaltenen Zahlen sind meist innerhalb einiger Procente zu niedrig. Das Amid und Anilid mussten wegen des abweichenden Verlaufs der Reaction von der vergleichenden Untersuchung ausgeschlossen werden.

In der Tabelle sind die Resultate der gleichartigen Versuche zusammengestellt. Die Zahlen geben in Procenten die Menge der angewandten Substanzen an, welche nach sechsstündiger Dauer der Reaction unter den von uns gewählten Versuchsbedingungen unverändert bleiben:

$\begin{array}{clr}\text { Zimmtsäure-Aethylester } & 6 \\ , & \text {-Methylamid } & 43 \\ " & \text { - Aethylamid } & 44 \\ " & \text {-Dimethylamid } & 29 \\ " & \text {-Diäthylamid } & 46 \\ " & \text {-Piperid } & 28 \\ " & \text { - Diphenylamid } & 32 .\end{array}$

Hieraus darf man folgern, dass der Säureester den Amiden an Reactionsfähigkeit bedeutend überlegen ist. Der basische Einfluss des Ammoniakrestes macht sich jedoch hierin nicht bemerkbar, denn grosse Unterschiede im Verhalten der Zimmtsäureamide gegen Malonsäureester bestehen nicht: Zimmtsäurepiperid nimmt den Ester kaum in geringerem Maasse auf als Zimmtsäurediphenylamid, trotz des sehr grossen Unterschiedes in der Stärke der Basen Piperidin und Diphenylamin.

Basische Reste in Nachbarstellung zum Carbonyl sind nicht im Stande, den sogenannten negativen Einfluss des Carbonyls der Stärke der Basen entsprechend abzuschwächen oder ganz aufzuheben.

Man kann die Carbonylwirkung auf dem eingeschlagenen Wege nicht bestimmen. Weder der erwartete Ausgleich zwischen dem negativen Carbonyl und dem positiven Rest, noch die verminderte Wirkung des Carbonyls auf die Kohlenstoffdoppelbindung ist bemerkbar. 
Die charakteristisehen Eigenschaften des Carbonyls sind demnach keine ausschliessliche Folge der sogenannten negativen Wirkung des Carbonyls, sondern beruhen auf anderen, noch unbekannten Eigenschaften. Unsere Vorstellungen von der negativen und positiven Natur der Radicale sind offenbar unzulänglich und fehlerhaft. Diese Ansicht, welche bereits von Ostwald, $\mathrm{Nef}^{20}$ ), Hantzsch ${ }^{21}$ ) und Anderen gelegentlich ausgesprochen wurde, findet eine weitere experimentelle Bestätigung durch Versuche mit aromatischen Glycinen, bei denen ein Ausgleich zwischen dem basischen Anilinrest und dem negativen Carbonyl an der Reactionsfähigkeit des Methylens zu erwarten,

$$
\left[\mathrm{C}_{6} \mathrm{H}_{5} \mathrm{NH}\right]-\mathrm{CH}_{2}-[\mathrm{CO}] \mathrm{OH},
$$

thatsïchlich aber nicht zu erkennen ist. Ich habe daher in der unten folgenden Abhandlung über die Natur der Radicale den Versuch gemacht, die Verhältnisse darzulegen, welche zwischen positiven, negativen, gesättigten und ungesättigten Radicalen bestehen.

\title{
Experimenteller Theil. \\ Ceber das Verhalten ungesätligter Verbindungen gegen Malonsäurcester ${ }^{22}$ );
}

\author{
bearbeitet von $P$. Hermann.
}

Die Versuche wurden in folgender Weise ausgeführt:

Man löst ein At. Natrium in der 20 fachen Menge absoluten Alkohols und giebt zu der abgekühlten Lösung ein Mol. Malonsäureäthylester, hierzu fügt man ein Mol. der ungesättigten Verhindung und kocht die Mischung sechs Stunden am Rückflusskühler im Wasserbade. Die so erhaltene Flüssigkeit soll kurz Reagenslösung heissen.

\footnotetext{
20) Diese Annalen $\mathbf{2 8 7}, 280$.

21) Ber. d. deutsch. chem. Ges. 32, 579, Amm.

22) Vergl. Chem. Centralbl. 1899, I, 730.
} 
Die weitere Aufarbeitung der Reactionsproducte musste eine verschiedene sein, je nach der Natur der ungesättigten Verbindungen. In den Fällen, wo es auf den Vergleich ankam, wie bei einigen Zimmtsäurederivaten, ist auch das Verfahren bei der Verseifung und Gewinnung unveränderter Substanzen das gleiche gewesen.

\section{Carbonylfreie ungesättigte Verbindungen.}

Styrol.

Die Mischung von Styrol $(3,5 \mathrm{~g})$ und Natriummalonsäureester erwärmt sich nicht. Während des Kochens scheiden sich Flocken von Metastyrol ab. Beim Abdestilliren der Hälfte des Alkohols geht ein Theil des unveränderten Styrols über. Der zurückbleibenden, mit Wasser und verdünnter Schwefelsäure versetzten Flüssigkeit entzieht Aether ein Gemenge von Malonsäureester und Styrol.

\section{Stilben.}

Der Kohlenwasserstoff krystallisirt unverändert beim Erkalten der heissen Mischung aus. Angewandt 2,5 g, wiedergewonnen $2,4 \mathrm{~g}$.

\section{Phenylnitroäthylen $\left.{ }^{23}\right)$.}

Nach dem Vermischen einer alkoholischen Lösung von PhenyInitroäthylen mit dem Natriummalonsäureester eutsteht alsbald ein weisser Niederschlag, welcher aus dem Natriumsalz des polymeren Phenylnitroäthylens ${ }^{24}$ ) besteht. Dieses erhält man beim Verreiben des Natriumsalzes mit verdünnter Schwefelsäure als amorphen Körper, der nach vorhergehender Bräunung und Zersetzung gegen $280^{\circ}$ schmilzt.

$0,1750 \mathrm{~g}$ gaben $0,4120 \mathrm{CO}_{2}$ and $0,0850 \mathrm{H}_{2} \mathrm{O}$.

$0,1720 \mathrm{~g} \quad, \quad 14,3 \mathrm{ccm}$ Stickgas bei $14^{\circ}$ und $765 \mathrm{~mm}$ Druck.

23) Darstellung nach Priebs, diese Amnalen 225, 320 .

${ }^{24)}$ Vergl. Nitrometastyrol, Blyth und Hofmann, diese Annalen 5*, 297. 


$\begin{array}{ccr} & \text { Berechnet fiir } & \text { Gefunden } \\ & \left(\mathrm{C}_{\mathrm{s}} \mathrm{H}_{7} \mathrm{NO}_{2}\right)_{\mathrm{x}} & \\ \mathrm{C} & 64,43 & 64,22 \\ \mathrm{H} & 4,70 & 5,39 \\ \mathrm{~N} & 9,40 & 9,88\end{array}$

Das Phenylnitroäthylen wird auch durch eine alkoholische Lösung von Natriumäthylat unter Wärmeentwickelung rasch polymerisirt.

\section{Diphenylbutadiën.}

Der Kohlenwasserstoff wurde nach der Vorschrift von Thiele ${ }^{2 \pi}$ ) dargestellt und unter verschiedenen Bedingungen mit Natriummalonester zusammengebracht ${ }^{26}$ ). Als nach dem üblichen Verfahren mit molekularen Mengen der Agentien keine Addition erfolgte (von $5 \mathrm{~g}$ Kohlenwasserstoff wiedergewommen $\pm g$ ), verwendeten wir einen grossen Ueberschuss von Natriummalonester in Benzolsuspension, und als auch dies nicht zum Ziele führte, ersetzten wir das Benzol durch Toluol, um die Reactionstemperatur zu steigern. Ein Additionsproduct haben wir nicht aufgefunden.

\section{Pyrrol und $N$-Phenylpyrrol ${ }^{\text {57)}) . ~}$}

Die Pyrrole verhielten sich gegen Natriummalonester und Acetessigester vollständig indifferent. Von $10 \mathrm{~g}$ Phenylpyrrol wurden $9,8 \mathrm{~g}$ unverändert aus der Reagenslösung abgeschieden.

\section{Benzalchinaldin ${ }^{28}$ ).}

Aus der mit dem $1 \frac{1}{2}$ fachen Volumen Wasser vermischten Reagenslösung scheidet sich unverändertes Benzalchinaldin aus, anfangs ölig, damn erstarrend. Das Benzalchinaldin ist in Gestalt des schwer löslichen, gelben Chlorhydrats leicht zu isoliren und wurde zur Identificirung in das bei $173^{\circ}$ schmelzende Di-

25) Diese Annalen 306, 198.

26) Versuche von G. Reinicke.

27) Kattnitz, Journ. f. pract. Chem. [2] 6, 148.

98) Wallach und Wüsten, Ber. d. deutsch. chem. Ges. 16, 2008. 
bromid verwandelt. Bei einem Versuche konnten $21,0 \mathrm{~g}$ von $23,2 \mathrm{~g}$ angewandter Substanz wiedergewonnen werden, bei einem anderen $17,5 \mathrm{~g}$ von $18,0 \mathrm{~g}$. Bei dem zweiten Versuche kam an Stelle der alkoholischen Lösung eine Suspension von Natriummalonsäureester in absolutem Aether zur Anwendung.

\section{Zimmtaldehydphenylhydrazon.}

Weder die zwischen den zwei Kohlenstoffatomen gelegene, noch die am Stickstoff stehende Doppelbindung nimmt den Malonester auf. Angewandt 11,0 g Hydrazon, wiedergewonnen $10,7 \mathrm{~g}$.

\section{Benzalacetonphenylhydrazon.}

Das Benzalaceton reagirt bekanntlich ausserordentlich leicht mit Natriummalonester. Beim Hydrazon des Ketons ist dies nicht der Fall. Angewandt $8,0 \mathrm{~g}$, wiedergewonnen $7,3 \mathrm{~g}$.

\section{Carbonylhaltige ungesättigte Verbindungen.}

Bei den folgenden Reactionen handelt es sich um Derivate der Zimmtsäure, Esster und Amide. Vor der Aufzählung der einzelnen Versuche muss beschrieben werden, in welcher Weise die Reagenslösung weiter verarbeitet wurde, um die Mengen der unverändert gebliebenen ungesättigten Verbindungen zu bestimmen. Die Mischung kann nach der Reaction die folgenden Verbindungen enthalten:

als unverändertes Ausgangsmaterial: Zimmtsäureester oder -amide, Malonsäureester,

als Reactionsproducte: den Ester der Phenylglutarcarbonsäure, einer Tricarbonsäure,

oder ein Amid derselben

$$
\mathrm{C}_{6} \mathrm{H}_{5}-\mathrm{CH}\left\langle\begin{array}{l}
\mathrm{CH}_{\mathrm{COOC}_{3} \mathrm{H}_{5}}^{\mathrm{COOC}_{2} \mathrm{H}_{5}} \\
\mathrm{COOO}_{2} \mathrm{H}_{\overline{3}}
\end{array}\right.
$$

$$
\mathrm{C}_{6} \mathrm{H}_{5}-\mathrm{CH}\left\langle\begin{array}{l}
\mathrm{CH}\left\langle\begin{array}{l}
\mathrm{COOC}_{2} \mathrm{H}_{5} \\
\mathrm{COOC}_{2} \mathrm{H}_{5}
\end{array}\right. \\
\mathrm{CH}_{2}-\mathrm{CO}-\mathrm{Nl}_{2}
\end{array} .\right.
$$


Die Ester sind in allen Fällen ölig oder niedrig schmelzend; die Isolirung derselben unterblieb. Die Verseifung wurde bei Zimmertemperatur bewirkt, da in der Wärme oft weitergehende Zersetzungen eintreten. Man versetzt die Reagenslösung mit einer concentrirten wässrigen Lösung eines grossen Ueberschusses von Kalihydrat, etwa dem vierfachen der für Malonester berechneten Menge. Falls der alkoholische und der wässrige Theil sich nicht völlig mischen, fügt man je nach Bedarf Alkohol oder Wasser hinzu, bis eine homogene Flüssigkeit entsteht, welche im verschlossenen Kolben mindestens zehn Tage stehen bleibt. Nach Verlauf dieser Zeit wird der Alkohol durch Abdunsten in flachen Schalen vollständig entfernt, wobei unveränderte, in kaltem Wasser wenig lösliche Amide sich krystallinisch abscheiden, zumal nach dem Verdünnen mit Wasser. Die Amide werden abfiltrirt, mit kaltem Wasser gewaschen, getrocknet und gewogen.

Das alkalische Filtrat enthält die durch Verseifung des Amids entstandene Zimmtsäure und die Phenylglutarcarbonsäure:

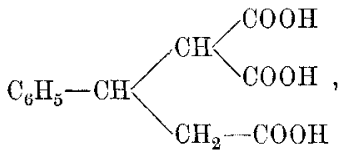

bezw. eine Amiddicarbonsäure:<smiles>NC(=O)CC(C(C(=O)O)C(=O)O)C(c1ccccc1)c1ccccc1</smiles>

Für die Trennung der Zimmtsäure von der Phenylglutarcarbonsäure ist von Bedeutung, dass die letztere als Derivat der Malonsäure in Wasser leicht löslich ist. Setzt man daher verdünnte Mineralsäuren bis zur Reaction auf Methylorange zur alkalischen Flüssigkeit, so wird die Zimmtsäure gefällt und kommt so zur Wägung. Die Phenylglutarcarbonsäure entzieht man der mineralsauren Flüssigkeit mit Aether. 
Die Amidcarbonsäuren sind mit einigen Ausnahmen in überschüssiger verdünnter Salzsäure löslich und dadurch von der Zimmtsäure zu trennen.

Abänderungen von diesem Gange sind erforderlich, wenn es gilt, das Endproduct, die Phenylglutarsäure, direct zu gewinnen unter Umgehung der Glutaramidearbonsäuren. Um dies zu erreichen, dampft man die mineralsaure Flüssigkeit auf dem Wasserbade zur Trockne ein und kocht den Rückstand 25 bis 30 Stunden mit Salzsäure (Siedep. $110^{\circ}$ ) unter Rückfluss. Nach dem Filtriren von etwas Harz krystallisirt aus der erkalteten und wenn nöthig eingeengten Flüssigkeit Phenylglutarsäure ${ }^{29}$ ). Die Gegenwart derselben beweist, dass eine Anlagerung eingetreten ist. Die Menge der so erhaltenen Säure bleibt natürlicherweise für die Beurtheilung des quantitativen Reactionsverlaufes unberücksichtigt.

Da es bei solchen Versuchen häufig nothwendig war, Zimmtsäure und Phenylglutarsäure von einander zu unterscheiden, so mag zur Charakteristik derselben eine Zusammenstellung der wichtigsten Eigenschaften dienen:

Schmelzpmint

Aequivalentgewiclit

Gehalt an $\left\{\begin{array}{l}\text { Kohleustoff } \\ \text { Wasserstoff }\end{array}\right.$
$\beta$-Phenylghtutarsäure

$138^{\circ}$

104

$63, \tilde{0}$

5,8

nicht reducirt

$86-87^{\circ}$
Zimmtsäuro

$183^{\circ}$

148

72,9

5,4

Kalte alkalische Permanganat-

losung wird

Schmelzpunkt des Methylesters

Zur vorläufigen Orientirung erwies sich das Verhalten gegen Permanganat und der Schmelzpunkt des Methylesters als sehr geeignet; zur sicheren Identificirung diente die Bestimmung des Aequivalentgewichtes und des Gehaltes an Kohlenstoff und Wasserstoff.

29) Ist Zimmtsänre und Phenylglutarsäure zugegen, so krystallisirt die Zimmtsäure znerst aus, dam folgt die Glutarsäure. Dicse kamn zuletzt der salzsauren Klïssigkeit anch mit Aether entzogen werden. 


\section{Zimmtsäureäthylester und Malonsäureester.}

Die Reagenslösung war aus $143 \mathrm{~g}$ Zimmtsäureäthylester, $128 \mathrm{~g}$ Malonsäureäthylester und $18,4 \mathrm{~g}$ Natrium bereitet worden und ergab nach zehnstündigem Kochen mit dem Doppelten der berechneten Menge Natronlauge, 9,0 g Zimmtsäure (d. i. $6 \mathrm{pC}$. der angewandten Zimmtsäure). Durch Extraction der mineralsauren Flüssigkeit mit Aether und durch Erhitzen des crbaltenen Oeles auf $150^{\circ}$ bis zur Beendigung der Kohlensäureabspaltung wurden $143 \mathrm{~g}$ nahezu reine Phenylglutarsäure gewonnen.

Um die Säure darzustellen, kann man auch statt der äquivalenten Menge Natrium nur $1 / 10$ derselben anwenden. Die Anlagerung volizieht sich dann ebenso glatt und die Menge der wiedergewonnenen Zimmtsäure wird geringer.

$$
\begin{gathered}
\beta \text {-Phenylglutarsäure, } \\
\mathrm{C}_{6} \mathrm{H}_{5}-\mathrm{CH} \backslash \\
\mathrm{CH}_{2}-\mathrm{COOH}
\end{gathered} .
$$

Die Säure ist bereits von Michael beschrieben worden ${ }^{30}$ ). Sie lässt sich durch Umkrystallisiren aus lrochendem Wasser oder aus Benzol, dem etwas Essigäther zugesetzt ist, oder durch Auskochen mit wenig Benzol, worin sie schwer lösich ist, reinigen. Man erbält sie so in rein weissen, prismatischen Tafeln. Sie ist in Wasser von Zimmertemperatur ziemlich löslich, schmilzt bei $138^{\circ}$ und wird in reinem Zustande durch alkalische Permanganatlösung erst nach längerem Stehen oxydirt.

Die elektrolytische Leitfühigkeit der Säure hat W. M e usel ${ }^{31}$ ) bestimmt. Das verwendete Präparat wurde von der Zimmtsäure durch Oxydation mit kalter alkalischer Permanganatlösung befreit und aus siedendem Wasser wiederholt umkrystallisirt.

\footnotetext{
30) Journ. f. pract. Chem. [9] 35, 352; Avery und Bouton, Amer. chem. .Journ. 20, 509; Chem. Centralb1. 1898, JI, 274.

31) Dissertation, Halle 1900.
} 


\begin{tabular}{|c|c|c|c|}
\hline \multicolumn{4}{|c|}{$\left.A_{\infty} 373 .^{32}\right)$} \\
\hline \multicolumn{4}{|c|}{ I. } \\
\hline $10^{-3} \varphi$ & $A$ & $100 \mathrm{a}$ & K \\
\hline 36,4 & 19,26 & 5,16 & 0,00772 \\
\hline 72,8 & 27,08 & 7,26 & 0,00763 \\
\hline 145,6 & 37,68 & 10,10 & 0,00779 \\
\hline 291,2 & 51,83 & 13,90 & 0,00770 \\
\hline \multicolumn{4}{|c|}{ II. } \\
\hline 45,6 & 21,53 & 5,77 & 0,00770 \\
\hline 91,2 & 29,97 & 8,03 & 0,00770 \\
\hline 182,4 & 41,76 & 11,19 & 0,00774 \\
\hline 364,8 & 56,99 & 15,28 & 0,00774 \\
\hline
\end{tabular}

Die specifische Leitfähigkeit des Wassers: 2,6.10 $10^{-6}$.

Das Ammoniumsalz der Säure erhält man beim Eingiessen einer concentrirten, absolut alkoholisehen Lösung der Säure in überschüssiges, absolut alkoholisches Ammoniak; aus der Mischung krystallisiren allmählich kleine, weisse, unregelmässig sechseckige Blättchen. So lange Spuren von Alkohol anhaften, ist das Salz an der Luft sehr zerfliesslich, sonst aber luftbeständig; es lässt sich aus Benzol umkrystallisiren. Beim Trocknen im Exsiccator verliert das Salz einen Theil des Ammoniaks, so dass die Analysen zu niedrig ausfielen (gefunden $13 \mathrm{pC}$. $\mathrm{NII}_{3}$, berechnet 14).

$$
\begin{gathered}
\text { Methylester, } \\
\mathrm{C}_{6} \mathrm{H}_{5}-\mathrm{OH} \\
\mathrm{CH}_{2}-\mathrm{COOCH}_{3}-\mathrm{COOCH}_{6}
\end{gathered} .
$$

Man kocht 5 g Phenylglutarsäure mit $30 \mathrm{ccm}$ Methylalliohol und $3 \mathrm{ccm}$ concentrirter Schwefelsäure fünf Stunden im Wasserbade. Die erkaltete Flüssigkeit wird auf Krystallsoda gegossen, wobei sich der Methylester krystallinisch abscheidet. Man erhält ihn so in fast theoretischer Ausbeute; er krystallisirt aus Methylalkohol und wenig Wasser in feinen, weissen Nadeln oder Prismen. Schmelzp. $86-87^{\circ}$.

92) Buchstabenbezeichung nach Kollyausch und Holborn: „Das Leitrermögen dex Elektrolyte"; vergl. anch diese Ammalen $\mathbf{3 0 8}, 186$. 
0,1525 g gaben $0,3660 \mathrm{CO}_{2}$ und $0,0938 \mathrm{H}_{2} \mathrm{O}$.

$\begin{array}{ccr} & \text { Berechnet für } & \text { Gefunden } \\ & \mathrm{C}_{13} \mathrm{H}_{18} \mathrm{O}_{\mathbf{4}} & \\ \mathrm{C} & 66,10 & 6 \tilde{,}, 45 \\ \mathrm{H} & 6,78 & 6,83\end{array}$

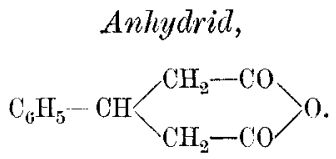

Man kocht Phenylglutarsäure fünf bis sechs Stunden mit etwa der fünffachen Menge Essigsäureanhydrid und destillirt dann die Essigsäure und das Essigsäureanhydrid bis $140^{\circ} \mathrm{ab}$. Das zurückbleibende Anhydrid der Phenylglutursäure erstarrt und wird durch Destillation im Vacuum oder durch Umkrystallisiren aus Benzol gereinigt. Es siedet bei 217-2190 unter $15 \mathrm{~mm}$ Druck und erstart in der Vorlage sofort zu einer fast weissen Krystallmasse. Schmelzpunkt nach dem Umkrystallisiren aus Benzol $10 \tilde{j}^{0}$.

$0,1760 \mathrm{~g}$ gaben $0,4455 \mathrm{CO}_{2}$ und $0,0892 \mathrm{H}_{2} \mathrm{O}$.

$\begin{array}{ccc} & \text { Berechnet fü̈r } & \text { Gefunden } \\ & C_{11} \mathrm{H}_{30} \mathrm{O}_{3} & \\ \mathrm{C} & \mathbf{6 9} & 69,47 \\ \mathrm{H} & 5,26 & 5,63\end{array}$

$0,1694 \mathrm{~g}$ Anhydrid wurden mit $52,0 \mathrm{ccm}$ Natronlauge vom Factor 0,065 unter Zusatz von etwas Alkohol fünf Stunden am Rïckflusskühler gekocht, die entstandene Lösung verbrauchte zu ihrer Neutralisation $22,6 \mathrm{ccm}$ Salpetersäure vom Factor 0,066 .

$\begin{array}{ccc} & \text { Berechnet fiur } & \text { Gefunden } \\ & \mathrm{C}_{11} \mathrm{H}_{10} \mathrm{O}_{3} & \\ \text { Leqgew. } & 95 & 90\end{array}$

Das Anhydrid ist leicht löslich in Chloroform, reichlich in Schwefelkohlenstoff and siedendem Benzol. Es wird von Ammoniak, Sodalösung und Natronlauge in der Kälte kaum aufgenommen. 
<smiles>CCCCCCC(=O)OCCCCC</smiles>

Phenylglutarsäure wird in einer kleinen, tubulirten Retorte geschmolzen, wobei sie unter Wasserabspaltung in Anhydrid überzugehen beginnt. In die geschmolzene Masse leitet man unter stärkerem Erhitzen trockues Ammoniakgas ein, bis das Ammoniak nicht mehr absorbirt wird und kein Wasser mehr überdestillirt. Der Inhalt der Retorte erstarrt bald und besteht aus dem Imid der Phenylglutarsäure. Dieses krystallisirt aus Weingeist oder Benzol in mikroskopischen Blättchen, aus heissem Wasser in weissen Flocken, bei langsamerem Erkalten in kleinen, zugespitzten Blättchen und schmilzt bei $173-174^{\circ}$. Es reagirt neutral und ist schwer löslich in Sodalösung, Ammoniak und verdünnter Salzsäure, löslich dagegen in kalter Natronlauge.

$0,1342 \mathrm{~g}$ gaben $0,3436 \mathrm{CO}_{2}$ und $0,0773 \mathrm{H}_{2} \mathrm{O}$.

$0,1816 \mathrm{~g} \quad, \quad 11,5 \mathrm{ccm}$ Stickgas bei $9,0^{\circ}$ und $755 \mathrm{~mm}$ Druck.

Berechmet fiir $\mathrm{C}_{11} \mathrm{H}_{11} \mathrm{O}_{2} \mathrm{~N}$

$\begin{array}{rrr}\mathrm{C} & \mathbf{6 9 , 8 4} & 69,84 \\ \mathrm{H} & \mathbf{5}, 82 & 6,40 \\ \mathrm{~N} & \mathbf{7 , 4 1} & 7,60\end{array}$

Phenylglutaranilsäure, $\mathrm{C}_{6} \mathrm{H}_{5}-\mathrm{CH}\left\langle\begin{array}{l}\mathrm{CH}_{2}-\mathrm{CO}-\mathrm{NHC}_{3} \mathrm{H}_{5} \\ \mathrm{CH}_{2}-\mathrm{COOH}\end{array}\right.$.

Bringt man Anilin zu einer Lösung des Anhydrids in Benzol, so wird unter Erwärmen eine ölige Abscheidung bemerkbar, welche sich bald an den Wänden des Gefüsses krystallinisch absetzt. Die entstandene Anilsäure ist löslich in Natriumcarbonat und krystallisirt aus Alkohol und etwas Wasser in feinen, rein weissen Nadeln; Schmelzp. $171^{\circ}$.

$0,1487 \mathrm{~g}$ gaben $0,3929 \mathrm{CO}_{2}$ und $0,0860 \mathrm{H}_{2} \mathrm{O}$.

$0,2391 \mathrm{~g} \quad, \quad 10,4 \mathrm{ccm}$ Stickgas bei $17,5^{\prime \prime}$ und $757 \mathrm{~mm}$ Druck. 
Vorländer, Kohlenstoffdoppelbindung und Carbonyl. 87

$\begin{array}{ccc} & \text { Berechnet für } & \text { Gefunden } \\ & \mathrm{C}_{17} \mathrm{H}_{17} \mathrm{O}_{3} \mathrm{~N} & \\ \mathrm{C} & 79,07 & 72,04 \\ \mathrm{H} & 6,01 & 6,42 \\ \mathrm{~N} & 4,95 & 5,04\end{array}$

$0,1972 \mathrm{~g}$ verbranchten zur Neutralisation 11,05 com Natronlange (Factor 0,065).

Bercchnet Gefunden

Aeqgew. $283 \quad 275$

\section{Zimmtsäureamide und Malonester.}

Die Zimmtsäureamide entstehen in guter Ausbeute, wenn man die entsprechenden Amine mit Zimmtsäurechlorid zusammenbringt. Bei der Darstellung des Diphenylamids werden beide Componenten in fester Form verwandt, sonst wird ein Mol. fein zerriebenes Chlorid zu zwei Mol. der Base (oder zu einem Mol. Base und einem Mol. Natriumhydroxyd), welche in Wasser gelöst sind, allmählich unter Kühlung und kräftigem Umschütteln zugesetzt. Das Reactionsproduct scheidet sich in fester Form aus und ist nach dem Auswaschen und Trocknen oft genïgend rein für die Versuche.

Die Ammoniakabkömmlinge der Zimmtsäure reagiren zwar nicht alkalisch, sie zeigen aber mit Ausnahme des Anilids und Diphenylamids noch basische Eigenschaften und lösen sich in verdünnter Salzsäure auf.

\section{Zimmtsäureamid.}

Das Amid schmilzt bei $145^{\circ}$. Bei der Reaction mit Natriummalonester tritt anfangs Lösung ein, aber schon nach etwa einhalbstündigem Kochen scheidet sich ein dicker Krystallbrei $\mathrm{ab}$, welcher aus dem Natriumsalz der Verbindung $\mathrm{C}_{14} \mathrm{H}_{15} \mathrm{O}_{4} \mathrm{~N}$ besteht ${ }^{33}$ ). Nach Zerlegung des mit warmem Wasser verriebenen Salzes (13,9 g aus 14,5 Amid) mit Essigsäure oder

33) In Folge eines Irrthums sind in der Anmerkung, diese Annalen 294, 298, Zimmtsäureamid und Anitid unter den Verbindungen angefiihrt, welche nicht mit Malonester reagiren. 
Kohlensäure krystallisirt die Verbindung aus kochendem Wasser in kleinen Nadeln und schmilzt bei $119^{\circ}$. Sie ist in Natronlauge, Ammoniak und überschüssiger Sodalösung löslich, wird durch verdünnte Salzsäure aus der alkalischen Lösung gefällt und vom Ueberschusse der Säure wieder gelöst; aus der salzsauren Lösung scheidet sie sich beim Stehen krystallinisch aus. Den Analysen zufolge ist die Verbindung aus Zimmtsäureamid und Malonester unter Austritt von Alkohol entstanden:<smiles>CCNC(=O)CC(C(=O)OCC)C1Oc2ccccc21</smiles>

l. $0,1265 \mathrm{~g}$ gaben $0,2975 \mathrm{CO}_{2}$ und $0,0686 \mathrm{H}_{2} \mathrm{O}$.

II. $0,1313 \mathrm{~g} \quad " \quad 0,3096 \quad \mathrm{CO}_{2} \quad, \quad 0,0702 \mathrm{H}_{2} \mathrm{O}$. $0,1371 \mathrm{~g} \quad, \quad 6,6 \mathrm{ccm}$ Stickgas bei $17,0^{\circ}$ und $750 \mathrm{~mm}$ Druck.

\begin{tabular}{cccc} 
& Berechnet für & \multicolumn{2}{c}{ Gefunden } \\
& $\mathrm{C}_{14} \mathrm{H}_{15} \mathrm{O}_{4} \mathrm{~N}$ & $\mathrm{I}$. & II. \\
$\mathrm{C}$ & 64,37 & 64,13 & 64,30 \\
$\mathrm{H}$ & 5,75 & 6,03 & 5,94 \\
$\mathrm{~N}$ & 5,36 & - & 5,53
\end{tabular}

Durch Spaltung mit kochender Salzsäure geht die Substanz in Phenylglutarsäure über, welche zur Identificirung titrirt und in den bei $87^{\circ}$ schmelzenden Dimethylester verwandelt wurde.

0,1225 g verbrauchten $24,0 \mathrm{ccm}$ Natronlauge (Factor 0,0494).

Berechnet für $\mathrm{C}_{11} \mathrm{H}_{12} \mathrm{O}_{4}$

Aeqgew. $\quad 104$
Gefunden

103

Das alkoholische Filtrat des bei der Reaction ansgefallenen Natriumsalzes enthielt neben Malonester $5,5 \mathrm{~g}$ mverändertes Zimmtsüureamid.

\section{Zimmtsäuremethylamid.}

Das Monomethylamid krystallisirt aus siedendem Wasser und ist leicht löslich in heissem Benzol und Alkohol; Schmelzpunkt $110-111^{0}$. 
Vorländer, Kohlenstoffdoppelbindung und Carbonyl. 89

$0,2871 \mathrm{~g}$ gaben $22,8 \mathrm{ccm}$ Stickgas bei $24, \tilde{\delta}^{\circ}$ und $759 \mathrm{~mm}$ Druck.

Berechnet fiir $\mathrm{C}_{10} \mathrm{H}_{11} \mathrm{NO}$

$\mathrm{N}$
Gefunden

8,91

Bei der Einwirkung von Malonsäureester blieben von $6,0 \mathrm{~g}$ angewandter Substanz 2,6 g unverändert, d. i. $43 \mathrm{pC}$.

Das Reactionsproduct wurde nicht isolirt, sondern sogleich mit Salzsäure verseift; erhalten $2,5 \mathrm{~g}$ Phenylglutarsäure.

$0,1726 \mathrm{~g}$ verbrauchten $25,23 \mathrm{ccm}$ Natronlange (Factor 0,065 ).

Berechnet für

$$
\mathrm{C}_{11} \mathrm{H}_{12} \mathrm{O}_{4}
$$

Leqgew. $\quad 104$
Gefunden

$10 \check{\jmath}$

\section{Zimmtsäuredimethylamid.}

Das Dimethylamid scheidet sich aus einer heissgesättigten wässrigen Lösung beim Erkalten in feinen Nädelchen oder irisirenden Blättchen aus. Schmelzp. $96^{\circ}$.

$0,2439 \mathrm{~g}$ gaben $17,4 \mathrm{ccm}$ Stickgas bei $19,5^{\circ}$ und $759 \mathrm{~mm}$ Drtuck.

$0,1841 \mathrm{~g} \quad, \quad 0,5079 \mathrm{CO}_{2}$ und $0,1304 \mathrm{H}_{2} \mathrm{O}$.

Berechnet für $\mathrm{C}_{11} \mathrm{H}_{13} \mathrm{ON}$

C $\quad 75,44$

$\mathrm{H} \quad 7,43$

$\mathrm{Y} \quad 8,00$
Gefunden

$7 \check{0}, 25$

7,87

8,21

Aus der Reagenslösung wurden nach der Verseifung mit Kalilauge von $8,8 \mathrm{~g}$ Dimethylamid $1,5 \mathrm{~g}$ unverändert wiedergewonnen und $0,9 \mathrm{~g}$ in Form von Zimmtsäure, d. i. zusammen 29 pC. der angewandten Substanz.

$0,0896 \mathrm{~g}$ verbrauchten $9,4 \mathrm{~cm}$ Natronlauge (Factor 0,065).

$\begin{array}{ccc} & \text { Berechnet } & \text { (ífunden } \\ \text { Aeqgew. } & 147 & 148\end{array}$

Nach der Spaltung mit Salzsäure erhielten wir 4,6 g Phenylglutarsäure.

$0,1135 \mathrm{~g}$ verbrauchten $17,5 \mathrm{ccm}$ Xatronlange (Factor 0,065).

Berechnet für $\mathrm{C}_{11} \mathrm{H}_{12} \mathrm{O}_{4}$

Aeqgerv. $\quad 104$
Gefunden

100 


\section{Zimmtsäureäthylamid.}

Das Monoäthylamid schmilzt nach dem Umkrystallisiren aus Wasser bei $92-93^{\circ}$.

0,1496 o gaben $0,4119 \mathrm{CO}_{2}$ und $0,1102 \mathrm{H}_{2} \mathrm{O}$.

$0,2227 \mathrm{~g} \quad, \quad 14,9 \mathrm{~cm}$ Stickgas bei $10,0^{\circ}$ und $763 \mathrm{~mm}$ Druck. Berechnet für Gefunden $\mathrm{C}_{11} \mathrm{H}_{13} \mathrm{NO}$

C $7 \check{5}, 44 \quad 75,08$

$\mathrm{H} \quad 7,43 \quad 8,15$

$\mathrm{N} \quad 8,00 \quad 8,06$

Nach der Behandlung der Reagenslösung mit kalter Kalilauge erwiesen sich von $17,0 \mathrm{~g}$ Aethylamid $7,5 \mathrm{~g}$ als unrerändert, d. i. 44 pC. Die durch Spaltung mit Salzsäure erhaltene Phenylglutarsäure wurde analysirt.

$0,1657 \mathrm{~g}$ gaben $0,3841 \mathrm{CO}_{2}$ und $0,0896 \mathrm{H}_{2} \mathrm{O}$.

$\begin{array}{ccc} & \text { Berechnet fiir } & \text { Gefunden } \\ & \mathrm{C}_{11} \mathrm{H}_{12} \mathrm{O}_{4} & \\ \mathrm{C} & 63,46 & 63,22 \\ \mathrm{H} & 5,77 & 6,00\end{array}$

$0,2781 \mathrm{~g}$ verbranchten $41,4 \mathrm{ccm}$ Natronlange (Factor 0,065 ).

Berechnet Gefunden

Aeqgew. $\quad 104 \quad 103$

\section{Zimmtsäurediäthylamid.}

Das Diäthylamid ist in Benzol und Weingeist sehr leicht löslich. Am besten krystallisirt man es aus kochendem Wasser und etwas Alkohol um, indem man den überschüssigen Alkohol wegkocht, bis sich die Lösung zu trüben beginnt. Beim Erkalten scheiden sich weisse Nadeln und Prismen aus. Schmelzpunkt $66^{\circ}$.

I. $0,1190 \mathrm{~g}$ gaben $0,3360 \mathrm{CO}_{2}$ und $0,0959 \mathrm{H}_{2} \mathrm{O}$.

$0,2689 \mathrm{~g} " 15,7 \mathrm{ccm}$ Stickgas bei $8,7^{\circ}$ und $745 \mathrm{~mm}$ Druck.

II. $0,1667 \mathrm{~g} \quad, \quad 10,0 \mathrm{ccm}$ Stickgas , $22,5^{0} \quad$, $763 \mathrm{~mm}$ Druck.

\begin{tabular}{cccc} 
& Berechnet für. & \multicolumn{2}{c}{ Gefunden } \\
& $\mathrm{C}_{13} \mathrm{H}_{13} \mathrm{ON}$ & I. & II. \\
$\mathrm{C}$ & 76,85 & 77,00 & - \\
$\mathrm{II}$ & 8,37 & 8,59 & - \\
$\mathrm{N}$ & 6,89 & 6,95 & 6,84
\end{tabular}


Mit einem Mol. Brom vereinigt sich das in Chloroform gelöste Diäthylamid loicht. Das Diäthylamid der Phenyldibrompropionsäure krystallisirt aus wässrigem Alkohol in schönen, weissen Prismen. Schmelzp. $127^{\circ}$.

I. $0,1724 \mathrm{~g}$ gaben $0,2666 \mathrm{CO}_{2}$ und $0,0786 \mathrm{H}_{2} \mathrm{O}$.

$0,2300 \mathrm{~g} \quad, \quad 7,9 \mathrm{ccm}$ Stickgas bei $9,5^{\circ}$ und $752 \mathrm{~mm}$ Iruck.

$0,1935 \mathrm{~g} \quad, \quad 0,2014 \mathrm{Ag} \cdot \mathrm{Br}$.

II. $0,1965 \mathrm{~g} \quad, \quad 7,3 \mathrm{ccm}$ Stickgas bei $14,5^{\circ}$ und $743 \mathrm{~mm}$ Drnck.

\begin{tabular}{lcrc} 
& Berechnet für & \multicolumn{2}{c}{ Gefunden } \\
\cline { 3 - 4 } & $\mathrm{C}_{13} \mathrm{H}_{17} \mathrm{NOBr}_{2}$ & I. & II. \\
$\mathrm{C}$ & 42,97 & 42,18 & - \\
$\mathrm{H}$ & 4,68 & 5,07 & - \\
$\mathrm{N}$ & 3,86 & 4,08 & 4,28 \\
$\mathrm{Br}$ & 44,08 & 44,29 & -
\end{tabular}

Bei der Anlagerung von Malonester wurden von $10,0 \mathrm{~g}$ Diäthylamid $4,6 \mathrm{~g}$ d. i. $46 \mathrm{pC}$. wiedergewonnen. Auf Zusatz von verdünnter Salzsäure zu der alkalischen Verseifungsflüssigkeit bildete sich eine zuerst ölige, dann krystallinische Fällung von Phenylglutardiäthylamidcarbonsäure $(7 \mathrm{~g})$, die bei etwa $145^{\circ}$ unter lebhafter Kohlensäureentwickelung schmilzt. Durch Spaltung derselben mit Salzsäure entsteht Phenylglutarsäure.

$0,1652 \mathrm{~g}$ gaben $0,3824 \mathrm{CO}_{2}$ und $0,0870 \mathrm{H}_{2} \mathrm{O}$.

$\begin{array}{ccr} & \text { Berechnet für } & \text { Gefunde } \\ & \mathrm{C}_{\mathbf{1 1}} \mathrm{H}_{12} \mathrm{O}_{4} & \\ \mathrm{C} & 63,46 & 63,13 \\ \mathrm{H} & 5,77 & 5,85\end{array}$

0,2714 g verbranchten $39,5 \mathrm{ccm}$ Natronlange (Factor 0,065).

Berechnet Gefunden.

Aeqgew. $\quad 106 \quad 104$

Zimmtsäurepiperid.

Herstein ${ }^{34}$ ) stellte das Piperid durch Verreiben ron einem Mol. Zimmtsäureanhydrid mit zwei Mol. Piperidin dar. Wir erhielten es durch Vermischen des in Benzol gelösten Zimmtsäurechlorids mit einer Lösung von Piperidin in Benzol.

34) Ber. d. deutsch. chem. Ges. 22, 2265. 
Die Verbindung wird aus wässrigem Methylalkohol umkrystallisirt und zeigt dann den Schmelzp. $122^{\circ}$.

$0,2663 \mathrm{~g}$ gaben $15,9 \mathrm{ccm}$ Stickgas bei $22,5^{\circ}$ und $760 \mathrm{~mm}$ Drtick. Berechnet für Gefunden $\mathrm{C}_{14} \mathrm{H}_{17} \mathrm{ON}$

$\mathrm{N} \quad 6,51 \quad 6,78$

Aus dem Piperid und Brom entsteht das aus Weingeist in langen Nadeln krystallisirende Piperid der Phenyldibrompropionsüure. Schmelzp. $189^{\circ}$ unter Zersetzung.

$0,2231 \mathrm{~g}$ gaben $0,3646 \mathrm{CO}_{2}$ und $0,0979 \mathrm{H}_{2} \mathrm{O}$.

$0,1618 \mathrm{~g} \quad, \quad \tilde{5}, 5 \mathrm{ccm}$ Stickgas bei $17,0^{\circ}$ und $750 \mathrm{~mm}$ Druck.

$0,2126 \mathrm{~g} \quad, \quad 0,2109 \mathrm{AgBr}$.

Berechnet für Geftuden $\mathrm{C}_{14} \mathrm{H}_{12} \mathrm{ONBr}_{2}$

C $44,80 \quad 44,57$

$\mathrm{H} \quad 4,53$

$\begin{array}{lll}\mathrm{N} & 3,73 & 3,91\end{array}$

$\mathrm{Br} \quad 42,67 \quad 42,21$

Versuche mit Malonsäureester:

1) Von 21,0 g Zimmtsäurepiperid wurden aus der Reagenslösung nach der Verseifung mit kalter Kalilauge 6,0 g unverändert wiedergewonnen, d. i. $28,5 \mathrm{pC}$.

2) Von $10,0 \mathrm{~g}$ angewandter Substanz $2,7 \mathrm{~g}$ d. i. $27 \mathrm{pC}$. Aus der alkalischen, vom unveränderten Piperid filtrirten Lösung fällt Salzsäure oder Schwefelsäure die Phenylglutarpiperidcarbonsäure aus:

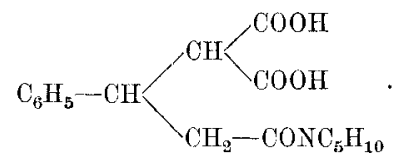

$0,1488 \mathrm{~g}$ gaben $0,3489 \mathrm{CO}_{2}$ und $0,0918 \mathrm{H}_{2} \mathrm{O}$.

$0,3093 \mathrm{~g}, \quad 12,5 \mathrm{ccm}$ Stickgas bei $14,0^{\prime \prime}$ und $760 \mathrm{~mm}$ Druck.

$\begin{array}{ccc} & \text { Berechnet für } & \text { Gefunden } \\ & \mathrm{C}_{12} \mathrm{H}_{21} \mathrm{O}_{5} \mathrm{~N} & \\ \mathrm{C} & 63,95 & 63,95 \\ \mathrm{H} & 6,58 & 6,85 \\ \mathrm{~N} & 4,40 & 4,75\end{array}$


Vorländer, Kohlenstoffdoppelbindung und Carbonyl. 93

$0,1786 \mathrm{~g}$ verbrauchten $17,2 \mathrm{ccm}$ Natronlauge (Factor 0,065 ).

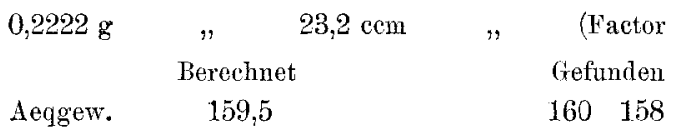

Die Säure krystallisirt aus kochendem Essigäther oder Wasser in weissen Prismen. Aus einer alkalischen Lösung wird sie durch verdünnte Salzsäure zunächst gefällt, dann durch einen Ueberschuss der Mineralsäure leicht gelöst und schliesslich beim Schütteln der sauren Lösung wieder krystallinisch ausgeschieden. Sie ist leicht löslich in Alkohol, wenig Iöslich in Benzol, Chloroform, Schwefelkohlenstoff, und schmilzt bei $146^{\circ}$ unter Kohlensäureabspaltung. Nach Beendigung der Gasentwickelung hinterbleibt ein $0 \mathrm{el}$, welches nach einiger Zeit erstarrt. Die so entstehende Säure

$$
\mathrm{C}_{6} \mathrm{H}_{5}-\mathrm{CH} / \begin{aligned}
& \mathrm{CH}_{2}-\mathrm{COOH} \\
& \mathrm{CH}_{2}-\mathrm{CONC}_{5} \mathrm{H}_{10}
\end{aligned}
$$

krystallisirt aus Wasser mit wenig Alkohol in weissen Blättchen. Schmelzp. $120^{\circ}$.

$0,1255 \mathrm{~g}$ verbrauchten $9,7 \mathrm{~cm}$ Natronlauge (Factor 0,0494 ).

$$
\begin{gathered}
\text { Berechnet fiir } \\
\mathrm{C}_{16} \mathrm{H}_{21} \mathrm{O}_{3} \mathrm{~N}
\end{gathered}
$$

Aeqgew.

275

262

Durch Kochen mit Salzsäure erhält man Phenylglutarsäure. $0,1834 \mathrm{~g}$ verbrauchten $28,4 \mathrm{ccm}$. Natronlauge (Factor 0,0607).

$\begin{array}{ccc} & \text { Berechnet } & \text { Gefunden } \\ \text { Aeqgew. } & 104 & 106\end{array}$

\section{Zimmtsäurediphenylamid.}

Das Diphenylamid ${ }^{35}$ ) stellten wir folgendermassen dar: 21,0 g Zimmtsäurechlorid and 42,3 g Diphenylamin, die durch Verreiben gemischt sind, werden in einer Porzellanschale auf dem Wasserbade zusammengeschmolzen, wobei die Reaction erfolgt. Die Schmelze wird mit kalter concentrirter Natronlauge verrieben, der Brei mit Wasser verdünnt und zur Entfernung

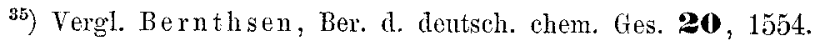




\section{Vorländer, Kohlenstoffdoppelbindung und Carbonyl.}

des Diphenylamins mit Aether geschüttelt; der ungelöste Rückstand $(31 \mathrm{~g})$ ist ziemlich reines Zimmtsäurediphenylamid. Der ätherische Auszug enthält nach dem Entfernen des Diphenylamins durch Einleiten von Chlorwasserstoff nur noch wenig des Körpers. Nach dem Umkrystallisiren aus Weingeist ist sein Schmelzp. $154^{\circ}$.

Bei dem Versuche mit Malonsäureester ist besonderer Werth darauf gelegt worden, dieselben Materialien, wie absoluten Alkohol, Malonester u.s.w. zu verwenden und unter denselben Bedingungen zu arbeiten, wie beim Zimmtsäurepiperid. Durch Verseifung mit Kalilauge gewinnt man zunächst die Gesammtmenge des imi angewandten Zimmtsäurediphenylamid $(21,0 \mathrm{~g})$ enthaltenen Diphenylamins $(11,5 \mathrm{~g}$ statt der berechneten $11,8 \mathrm{~g})$. Aus der alkalischen Flüssigkeit fällt verdünnte Schwefelsäure 3,3 g Zimmtsäure, d. i. $32 \mathrm{pC}$.

Das Filtrat von der Zimmtsäure wurde mit Aether ansgeschüttelt. Dieser hinterlies $5,5 \mathrm{~g}$ der syrupösen Phenylglutarcarbonsäure, die sich leicht in Alkohol, Benzol und warmem Wasser löst. Die alkoholische Lösung wird durch Wasser nicht gefällt, und die Säure bleibt beim langsamen Verdunsten des Lösungsmittels ölig zurück. Sie verliert beim Erhitzen über $120^{\circ}$ Kohlensäure und der Rückstand besteht aus Phenylglutarsäure.

$0,1897 \mathrm{~g}$ verbrauchten $27,6 \mathrm{~cm}$ Natronlange (Factor 0,065 ).

$\begin{array}{ccc} & \text { Berechnet } & \text { Gefinden } \\ \text { Aeqgew. } & 104 & 106\end{array}$

\section{Zimmtsüureanilid.}

Die Anlagerung von Malonester an Zimmtanilid geht nicht in so einfacher Weise vor sich, wie bei den oben beschriebenen Zimmtsäurederivaten. Die Reagenslösung enthält ausser der Mischung von unverändertem Ausgangsmaterial und Reactionsproduct einige andere Körper, welche weiterhin theils durch Verseifung, theils durch Condensation aus dem primären Reactionsproducte 
Vorländer, Kohlenstoffdoppelbindung und Carbonyl. 95

hervorgegangen sind.

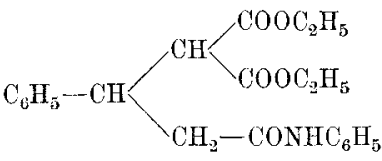

Während des Kochens der Reagenslösung scheidet sich ein weisser Niederschlag (A) aus, und dieser wird zunächst vom alkoholischen Filtrat (B) getrennt.

A. Der Niederschlag, dessen Gewicht bei einem Versuche $110 \mathrm{pC}$, bei einem zweiten $130 \mathrm{pC}$. vom angewandten Zimmtanilid betrug, besteht aus einem Gemenge von Phenylghutaranilester, $\mathrm{C}_{20} \mathrm{H}_{19} \mathrm{O}_{4} \mathrm{~N}$ (I)

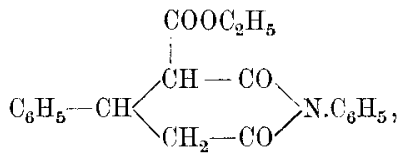

und dem Natriumsalz einer Säure (II), welche im reinen $\mathrm{Zu}$ stande nicht darzustellen war. Sie ist vermuthlich durch theilweise Verseifung entstanden:<smiles>CCCC(C=CC(C(=O)O)c1ccccc1)C(=O)OCC</smiles>

Der Anilester bleibt beim Verreiben und Erwärmen des Niederschlags A mit Wasser unter Einleiten von Kohlendioxyd zurück (etwa 25 pC. vom Gewicht des Niederschlags), das Natriumsalz der Säure geht in Lösung.

I. Der Anilester $\mathrm{C}_{20} \mathrm{H}_{19} \mathrm{O}_{4} \mathrm{~N}$ krystallisirt aus kochendem Weingeist in weissen Nädelchen und schmilzt bei $166^{\circ}$; er ist in Sodalösung und Ammoniak unlöslich.

I. $0,1904 \mathrm{~g}$ gaben $0,4948 \mathrm{CO}_{2}$ und $0,9060 \mathrm{H}_{2} \mathrm{O}$.

$0,3372 \mathrm{~g} \quad, \quad 11,2 \mathrm{ccm}$ Stickgas bei $4,5^{\circ}$ und $773 \mathrm{~mm}$ Druck.

II. $0,1689 \mathrm{~g} \quad, \quad 0,4380 \mathrm{CO}_{2}$ und $0,0902 \mathrm{H}_{2} \mathrm{O}$.

$0,1673 \mathrm{~g} \quad, \quad 6,0 \mathrm{ccm}$ Stickgas bei $9,0^{\circ}$ and $763 \mathrm{~mm}$ Druck.

III. $0,1704 \mathrm{~g} \quad, \quad 0,4427 \mathrm{CO}_{2}$ und $0,0940 \mathrm{H}_{2} \mathrm{O}$.

$0,1975 \mathrm{~g}, \quad 7,4 \mathrm{ccm}$ Stickgas bei $9,0^{\circ}$ und $755 \mathrm{~mm}$ Druck. 
96 Vorländer, Kohlenstoffdoppelbindung und Carbonyl.

\begin{tabular}{ccrcc} 
& Berechmet füir & \multicolumn{3}{c}{ Gefunden } \\
\cline { 3 - 5 } & $\mathrm{C}_{\mathrm{a} 0} \mathrm{H}_{19} \mathrm{O}_{4} \mathrm{~N}$ & I. & II. & III. \\
$\mathrm{C}$ & 71,22 & 70,89 & 70,74 & 70,86 \\
$\mathrm{H}$ & 5,64 & 5,29 & 5,93 & 6,13 \\
$\mathrm{~N}$ & 4,16 & 4,15 & 4,34 & 4,48
\end{tabular}

Die Natur des Körpers ergiebt sich aus der Spaltung mit kochender Salzsäure, wobei neben Anilin Phenylglutarsäure entsteht,

0,2370 g erforderten $35,35 \mathrm{ccm}$ Natronlange (Factor 0,065).

$\begin{array}{ccc} & \text { Berechnet } & \text { Gefunden } \\ \text { Aeqgew. } & 104 & 103\end{array}$

und aus der Verseifung mit kalter alkoholischer Kalilauge. Aus der vom Alkohol befreiten Lauge fällt verdünnte Schwefelsäure eine anfangs ölige, dann erstarrende Säure aus, welche aus einem Gemisch von Alkohol und Wasser in Nädelchen krystallisirend zu erhalten ist. Man darf dieselbe wohl als die Anildicarbonsäure

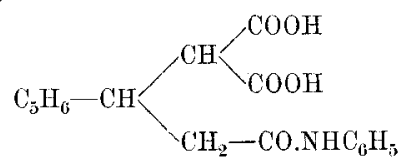

betrachten; sie giebt beim Erhitzen Kohlensäure $a b$ und der Rückstand besteht aus der bereits beschriebenen Phenylglutaranilsäure. Schmelzp. $169^{\circ}$.

$$
\mathrm{C}_{6} \mathrm{H}_{5}-\mathrm{CH}\left\langle\begin{array}{l}
\mathrm{CH}_{2}-\mathrm{COOH} \\
\mathrm{CH}_{2}-\mathrm{CONHC} \mathrm{NH}_{5}
\end{array} .\right.
$$

$0,1381 \mathrm{~g}$ gaben $0,3662 \mathrm{CO}_{2}$ und $0,0786 \mathrm{II}_{2} \mathrm{O}$.

\begin{tabular}{ccc}
$0,2173 \mathrm{~g}$ & \multicolumn{2}{c}{$9,2 \mathrm{ccm}$ Stickgas bei $9,5^{\circ}$ und $762 \mathrm{~mm}$ Druck. } \\
& $\begin{array}{c}\text { Berechnet für } \\
\text { Gefunden }\end{array}$ \\
$\mathrm{C}$ & $\mathrm{C}_{17} \mathrm{H}_{17} \mathrm{O}_{3} \mathrm{~N}$ & \\
$\mathrm{H}$ & 72,07 & 79,31 \\
$\mathrm{~N}$ & 6,01 & 6,32 \\
& 4,95 & 5,11
\end{tabular}

II. Aus der vom Phenylglutaranilester abfiltrirten Lösung des Natriumsalzes, welche schwach alkalisch reagirt, fällt verdünnte Schwefelsäure die erwähnte Säure aus in Form har- 
ziger, allmäblich fester werdender Klumpen (etwa $60 \mathrm{pC}$. vom Gewicht des Niederschlages A). Dieser Körper ist nur wenig gefärbt, aber es gelingt nur schwierig, ihn aus Wasser und wenig Alkohol in krystallinischer Form zu erhalten: mikrosskopische Nadeln, Schmelzpunkt um $85-90^{\circ}$ unter Kohlensäureentwickelung.

Durch Spaltung mit kochender Salzsäure gingen aus $8,0 \mathrm{~g}$ neben Anilin 4,1 g Phenylglutarsäure hervor.

$0,1611 \mathrm{~g}$ gaben $0,3719 \mathrm{CO}_{2}$ und $0,0872 \mathrm{H}_{2} \mathrm{O}$.

$\begin{array}{ccc} & \text { Berechnet für } & \text { Gefunden } \\ & \mathrm{C}_{11} \mathrm{H}_{12} \mathrm{O}_{4} & \\ \mathrm{C} & 63,46 & 63,01 \\ \mathrm{H} & 5,77 & 6,01\end{array}$

$0,3047 \mathrm{~g}$ verbrauchten $44,88 \mathrm{ccm}$ Natronlauge (Factor 0,065 ).

Berechnet Gefunden

Aeqgew. $\quad 104 \quad 104$

Durch Spaltung mit kalter alkoholischer Kalilauge wurde wie oben die Anildicarbonsäure erbalten ( $8 \mathrm{~g}$ aus $12 \mathrm{~g}$ Substanz), welche sich in heissem Wasser löst und beim Erkalten nach einiger Zeit in Büscheln und Sternen mikroskopischer Nadeln auskrystallisirt. Schmelzp. $153^{\circ}$ unter Kohlensäureentwickelung. $0,1908 \mathrm{~g}$ gaben $7,2 \mathrm{ccm}$ Stickgas bei $18,5^{0}$ und $749 \mathrm{~mm}$ Druck.

$$
\begin{array}{ccc} 
& \text { Berechnet für } & \text { Gefunden } \\
& \mathrm{C}_{17} \mathrm{H}_{18} \mathrm{O}_{5} \mathrm{~N} & \\
\mathrm{~N} & 4,28 & 4,30
\end{array}
$$

$0,1494 \mathrm{~g}$ erforderten $18,7 \mathrm{cem}$ Natronlauge (Factor 0,0494).

Berechnet Gefunden

Aeqgew. $\quad 163,5 \quad 162$

Schliesslich ergab die Anildicarbonsäure beim Erhitzen über den Schmelzpunkt Phenylglutaranilsäure.

$0,1694 \mathrm{~g}$ gaben $0,4472 \mathrm{CO}_{2}$ und $0,0956 \mathrm{H}_{2} \mathrm{O}$.

$0,2064 \mathrm{~g} \quad, \quad 9,3 \mathrm{ccm}$ Stickgas bei $18,0^{\circ}$ und $749 \mathrm{~mm}$ Druck.

$\begin{array}{ccr} & \text { Berechnet für } & \text { Gefunde } \\ & \mathrm{C}_{37} \mathrm{H}_{17} \mathrm{O}_{3} \mathrm{~N} & \\ \mathrm{C} & 72,07 & 71,96 \\ \mathrm{H} & 6,01 & 6,27 \\ \mathrm{~N} & 4,95 & 5,15\end{array}$

Anualen der Chemie 320. Bd. 
B. Das alkoholische Filtrat vom Niederschlage $A$ blieb mit überschüssiger Kalilange 20 Tage stehen und es wurde in der gewöhnlichen Weise das unveränderte Zimmtanilid (29 pC. der angewandten Menge) abgeschieden. Aus der alkalischen Flüssigkeit fällt verdünnte Salzsäure eine Säure in Flocken aus, welche aus wässrigem Alkohol in kleinen, weissen Prismen krystallisirt und bei $150-152^{0}$ unter Kohlensäureentwickelung schmilzt. Wider Erwarten war dieser Körper kein Derivat der Phenylglutarsäure, sondern der Zimmtsäure, welche durch Spaltung vermittelst Salzsäure mit allen ihren charakteristischen Eigenschaften gewonnen wurde.

\section{Zimmtsäurenitril und Malonsäureester.}

Es war zu erwarten, dass das Zimmtsäurenitril sich wie eine carbonylhaltige Substanz verhalten und mit Natriummalonester in Verbindung treten würde. Das dicke Oel, welches nach dem Abdampfen der alkoholischen Reagenslösung zurückbleibt, wurde mit rauchender Salzsäure etwa 24 Stunden im Rohre auf $100^{\circ}$ erhitzt, um den Nitrilester

$$
\mathrm{C}_{6} \mathrm{H}_{5}-\mathrm{CH}\left\langle\begin{array}{l}
\mathrm{CH}\left(\mathrm{COOCO}_{2} \mathrm{H}_{5}\right)_{2} \\
\mathrm{CH}_{2}-\mathrm{CN}
\end{array}\right.
$$

zu verseifen. In der salzsauren Flüssigkeit befand sich neben wenig Zimmtsäure und Chlorammonium die gesuchte Phenylglutarsäure als Hauptreactionsproduct.

$0,1931 \mathrm{~g}$ gaben $0,4521 \mathrm{CO}_{2}$ und $0,1030 \mathrm{H}_{2} \mathrm{O}$.

$\begin{array}{ccc} & \text { Berechnet fïr } & \text { Gefunden } \\ & \mathrm{C}_{11} \mathrm{H}_{12} \mathrm{O}_{4} & \\ \mathrm{C} & 63,46 & 63,79 \\ \mathrm{H} & 5,77 & 5,90\end{array}$

0,2803 g erforderten 44,7 cem Natronlauge (Factor 0,0608).

Berechnet Gefunden

Aeqgew. $\quad 104 \quad 103$ 